\title{
Response to "Alcohol and COVID-19: How Do We Respond to This Growing Public Health Crisis?"
}

$\mathrm{J}$ Gen Intern Med 36(8):2476

DOI: $10.1007 /$ s11606-021-06824-3

(C) Society of General Internal Medicine 2021

$\mathrm{T}_{\mathrm{w}}$ o the Editor;

We read with interest "Alcohol and COVID-19: How Do We Respond to This Growing Public Health Crisis?" noting particularly how COVID-19 is distinct from other global tragedies. ${ }^{1}$ We appreciate the authors' recognition of the persistent barriers in primary care to treating patients with alcohol use disorders, and careful description of how to improve treatment. Additionally, it is not uncommon for a hospitalist to be the first clinician the patient has contact with, especially if the patient is not established with primary care. This puts hospitalists in a unique position to have an impact on treating patients with alcohol use disorders. As hospitalists engaged in improving the care and clinical experience of people with alcohol use disorders, we are writing to implore inpatient clinicians to also develop processes and systems to connect patients with treatment.

A review published in 2018 examined data on medications for alcohol use disorder and concluded that naltrexone, acamprosate, and topiramate are the most effective in reducing alcohol use, but are notably underutilized. ${ }^{2}$ Additional support for prescribing naltrexone was demonstrated earlier this year in a real-world study finding that this medication, either alone or in combination with disulfiram or acamprosate, was associated with lower risk of hospitalization due to any cause, including alcohol-related. ${ }^{3}$

Hospitalists can have an important role in promoting the use of medications for patients with alcohol use disorders. In 2015, an intervention to identify patients who may benefit from alcohol use disorder treatment increased prescriptions of naltrexone while decreasing readmissions and emergency department visits. ${ }^{4}$ Furthermore, a 2018 study evaluating the effects of clinician education on naltrexone and the adoption of algorithms for evaluating patients for naltrexone resulted in subgroups of patients having reduced emergency department visits and rehospitalizations. 5

Received February 9, 2021

Accepted April 9, 2021

Published online April 26, 2021
We believe hospital medicine teams should take advantage of opportunities to improve care of patients with alcohol use disorders. Hospitalization is an opportune time for a patient to receive screening, counseling, and treatment of alcohol use disorders. Hospitalists are well-positioned to promote solutions to public health crises, and have a history of doing so-particularly among patients who are not regularly seen in primary care. Initiating treatment for alcohol use disorders while inpatient or at the time of discharge, and providing warm hand-offs to colleagues in primary care, is the next step in improving care among this vulnerable population of patients.

Eileen Barrett, MD, MPH, SFHM, MACP ${ }^{1}$

Krystle Apodaca, DNP, FHM ${ }^{1}$

${ }^{1}$ Department of Internal Medicine, University of New Mexico,

Albuquerque, NM, USA

Corresponding Author: Krystle Apodaca, DNP, FHM; Department of Internal Medicine, University of New Mexico, Albuquerque, NM, USA (e-mail: ).

Declarations:

Conflict of Interest: Neither Dr Apodaca nor Dr Barrett have conflicts of interest.

\section{REFERENCES}

1. Sugarman, DE, Greenfield, SF. Alcohol and COVID-19: How Do We Respond to This Growing Public Health Crisis?. J Gen Intern Med 36, 214-215 (2021). https://doi.org/10.1007/s11606-020-06321-Z

2. Kranzler HR, Soyka M. Diagnosis and Pharmacotherapy of Alcohol Use Disorder: a Review. JAMA. 2018;320(8):815-824. https://doi.org/10. 1001/jama.2018.11406

3. Milja Heikkinen, M, Taipale, H, Tanskanen, A, Mittendorfer-Rutz, E, et al. Real-world Effectiveness of Pharmacological Treatments of Alcohol Use Disorders in a Swedish Nationwide Cohort of 125,556 patients. Addiction 2021. https://doi.org/10.1111/add. 15384

4. Wei, J, Defries, T, Lozada, M, et al. An Inpatient Treatment and Discharge Planning Protocol for Alcohol Dependence: Efficacy in Reducing 30-Day Readmissions and Emergency Department Visits. J Gen Intern Med 30, 365-370 (2015). https://doi.org/10.1007/s11606-014-2968-9

5. Stephens JR, Moore, C, Stepanek, KV, et al. Implementation of a Process for Initiating Naltrexone in Patients Hospitalized for Alcohol Detoxification or Withdrawal. J Hosp Med. 2018;4;221-228. https://doi.org/10.12788/ jhm.2900

Publisher's Note: Springer Nature remains neutral with regard to jurisdictional claims in published maps and institutional affiliations. 